\title{
An exploratory study on the synthesis of heparin-like oligosaccharides by polycondensation
}

\author{
Niels-Christian Reichardt and Manuel Martín-Lomas* \\ Grupo de Carbohidratos, Instituto de Investigaciones Químicas, CSIC, Américo Vespucio s/n, \\ Isla de La Cartuja, 41092 Sevilla, Spain \\ E-mail: manuel.martin-lomas@iiq.csic.es
}

\section{Dedicated to Professors José Elguero and Pedro Molina on the occasion of their $70^{\text {th }}$ and $60^{\text {th }}$ birthdays, respectively}

(received 22 Dec 04; accepted 17 Feb 05; published on the web 11 Mar 05)

\begin{abstract}
The synthesis of heparin oligosaccharide fragments by autocondensation of suitably protected Dglucosamine $\alpha 1 \rightarrow 4$ L-iduronic acid disaccharide constructs containing both glycosyl- donor and glycosyl- acceptor functionalities has been explored. Trichloroacetimidate and n-pentenyl groups have been investigated for anomeric activation. The generation of building blocks equipped with a trichloroacetimidate function is seriously hampered by the presence of the free- $\mathrm{OH}$ glycosylacceptor group on the same molecule, which favors an intramolecular transesterification reaction. Using an n-pentenyl leaving group, the glycosylation promoter competes as a nucleophile with the $\mathrm{OH}$ glycosyl acceptor in the autocondensation process, giving rise to fast termination of the chain reaction, a low yield, and low degree of polymerization. It is concluded that, in this area, polycondensation can hardly compete with elaborate stepwise approaches as a result of the intrinsic low reactivity — both as a glycosyl donor and as glycosyl acceptor — of the D-glucosamine $\alpha 1 \rightarrow 4$ L-iduronic acid building blocks.
\end{abstract}

Keywords: Heparin oligosaccharides, synthesis, polycondensation, trichloroacetimidate, npentenyl glycosides

\section{Introduction}

As part of a program on the molecular basis of the activation of fibroblast growth factors (FGFs) by glycosaminoglycans (GAGs) we have developed a modular approach for a completely stereoselective block synthesis of oligosaccharides containing the repeating unit (D-glucosamine $\alpha 1 \rightarrow 4$ L-iduronate) of the major sequence of heparin. ${ }^{1}$ This approach, which has been extended successfully to solid-phase synthesis using an acceptor-bound strategy, ${ }^{2}$ has allowed the 
preparation of several hexa- and octa-saccharides with different charge distributions and orientations ${ }^{3}$ which have been used for structure-activity studies. ${ }^{4}$ FGFs ${ }^{5}$ constitute a family of polypeptides belonging to the class of heparin- binding proteins. ${ }^{6}$ These heparin- binding proteins ${ }^{6}$ are involved in important cellular functions and their biological activities are tightly regulated by heparan sulfate GAGs. ${ }^{7}$ Because of the intrinsic heterogeneity of GAGs, ${ }^{7}$ the synthesis of heparin oligosaccharide fragments with defined size, sequence, and charge distribution is essential for understanding the GAG-protein molecular recognition phenomena in living systems. The size and the sequence of the oligosaccharide fragment, and the distribution and orientation of sulfate groups along the oligosaccharide chain, are thought to play a key role in these recognition events. ${ }^{6}$

Effective as our reported block synthesis may be, the preparation of heparin fragments for interaction studies requires the development of new methods to speed up and simplify the process. In this paper we explore the scope and the limitations of a polycondensation reaction of conveniently protected building blocks to construct the oligosaccharide skeleton in a single polymerization step.

\section{Results and Discussion}

Our block synthesis of heparin oligosaccharide fragments is based on the stereoselective assembly of disaccharide building blocks with protecting group patterns designed to achieve a defined distribution of sulfate groups in the final product. ${ }^{1-3}$ We thought that a polycondensation reaction of a properly selected disaccharide building block as the repeating unit would represent an attractive alternative to the stepwise synthesis for the preparation of regular sequences. The synthesis of polysaccharides using a controlled chemical polymerization has been attempted previously with success in a few cases, ${ }^{8}$ and the enzymatic polymerization of a disaccharide building block has been used to synthesize a fragment of the GAG hyaluronan. ${ }^{9}$ Cationic ringopening-polymerization has been reported from 1,2-ortho-esters ${ }^{10}$ and 1,6-anhydrohexoses, ${ }^{8 \mathrm{c}}$ and polycondensation reactions have been described from 1,2-cyanoethylene derivatives. ${ }^{8 \mathrm{a}} \mathrm{We}$ envisaged the possibility of generating homogeneously protected heparin oligosaccharide fragments by autocondensation of a suitably protected disaccharide repeating unit as indicated in Chart 1.

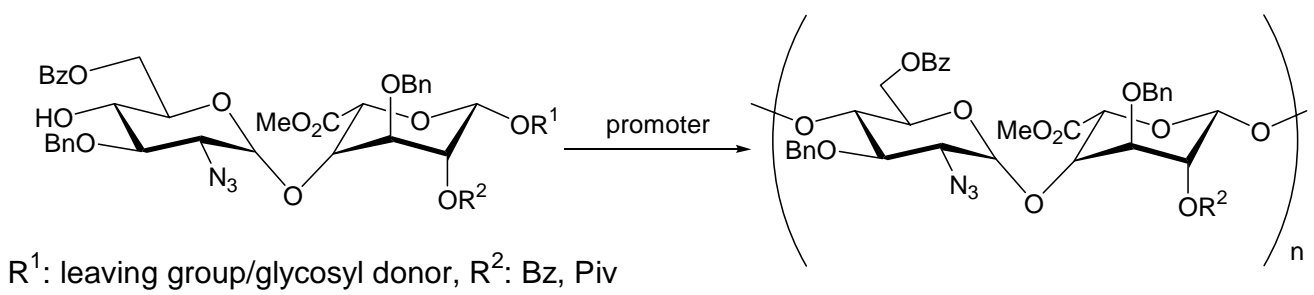

Chart 1. Principle of proposed autocondensation reaction. 
For their proven efficiency in our stepwise synthesis of heparin oligosaccharides ${ }^{1-3}$ we first used trichloroacetimidates as leaving groups. Thus, the preparation of a bifunctional trichloroacetimidate carrying both glycosyl-donor and glycosyl-acceptor functionalities was attempted from disaccharide $\mathbf{1}^{1,3 a}$ which is currently used in our stepwise syntheses. Compound $\mathbf{1}$ was converted into $\mathbf{2}$ following a well established sequence, ${ }^{1,3 a}$ and $\mathbf{2}$ was desilylated ${ }^{11}$ to give the diol 3 (Scheme 1).
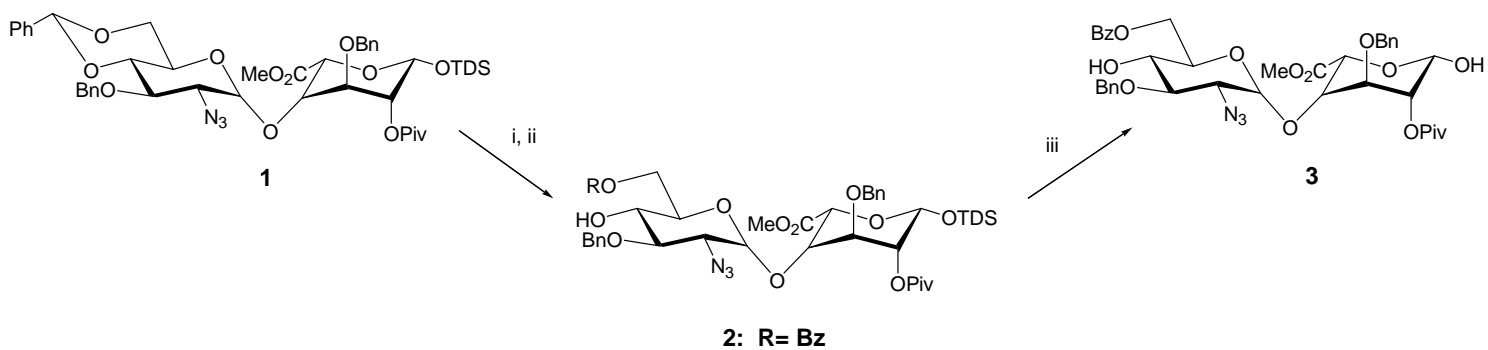

Scheme 1. Reagents and conditions: (i) EtSH, p-TsOH, $\mathrm{CH}_{2} \mathrm{Cl}_{2}$; (ii) $\mathrm{BzCN}, \mathrm{Et}_{3} \mathrm{~N}, \mathrm{CH}_{3} \mathrm{CN}, 92 \%$; (iii) $\mathrm{HF}:$ pyr, $\mathrm{THF}, 4^{\circ} \mathrm{C}, 94 \%$.

Attempted selective installation of the trichloroacetimidate group at the anomeric position of diol 3 by treatment with a slight excess of trichloroacetonitrile in the presence of $\mathrm{K}_{2} \mathrm{CO}_{3}{ }^{12}$ afforded lactone $\mathbf{4}$ as the main product (Scheme 2). When this reaction was performed with excess of reagent a mixture of $\mathbf{4}$ and the trichloroacetimidate $\mathbf{5}$ was formed. The unexpected formation of 4 by intramolecular transesterification of the methyl ester group with the free anomeric hydroxyl group appears to be a consequence of the presence of the free 4'-OH in $\mathbf{3}$, since this reaction has never before been observed in trichloroacetimidate formation in this series. In this reaction, which involves a conformational inversion of the L-iduronic acid pyranoid ring followed by the base- promoted transesterification of the methyl ester, the 4'-OH may be involved in the activation of the ester function through a hydrogen bridge. In any case, these results demonstrated that trichloroacetimidate anomeric activation is not suitable for straightforward generation of the disaccharide repeating unit and other activation methods had to be investigated.

We then turned our attention to n-pentenyl activation as pentenyl glycosides ${ }^{13}$ had been reported to be effective glycosylating agents in the coupling of L-iduronic acid derivatives to the 4-OH group of D-glucosamine units. ${ }^{14}$ However, the installation of the n-pentenyl group at the anomeric position of a L-iduronic acid derivative has proven to be feasible only after previously activating that position as a bromide or a trichloroacetimidate. ${ }^{15}$ Therefore, the known trichloroacetimidate $6^{3 \mathrm{c}}$ was reacted ${ }^{16}$ with excess pent-4-en-1-ol to give the n-pentenyl glycoside 7 in $62 \%$ yield (Scheme 3 ). 


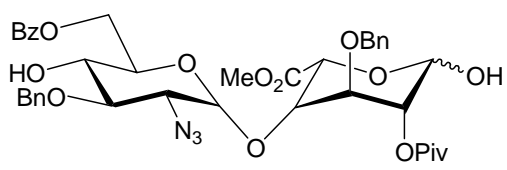

3
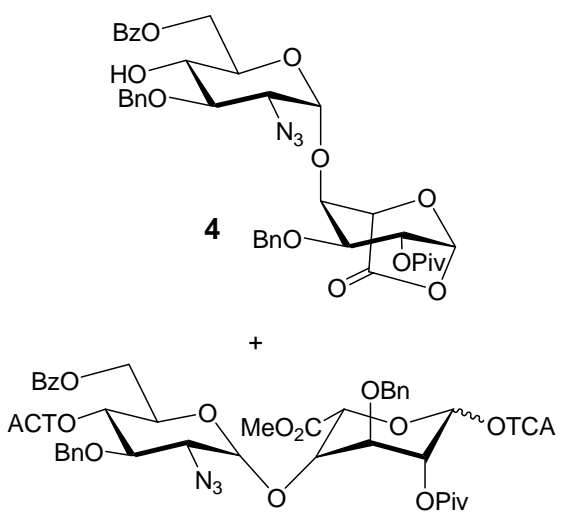

5

Scheme 2. (i) $\mathrm{K}_{2} \mathrm{CO}_{3}, \mathrm{Cl}_{3} \mathrm{CN}, \mathrm{CH}_{2} \mathrm{Cl}_{2}$.

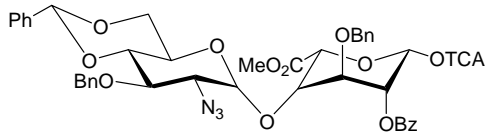

6
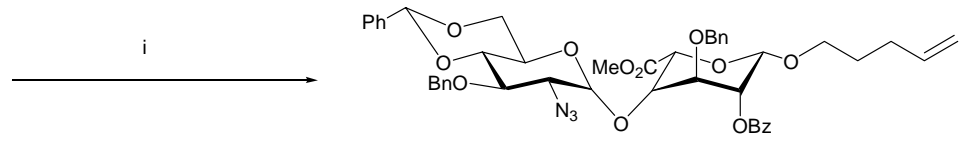

Scheme 3. (i) 4-penten-1-ol, TMSOTf, $\mathrm{CH}_{2} \mathrm{Cl}_{2}, 47 \%$.

Compound 7 was transformed into the bifunctional building block 9 by trans-acetalation of the benzylidene acetal ${ }^{17}$ to give diol $\mathbf{8}$ followed by selective benzoylation ${ }^{18}$ of the primary hydroxyl group in 8 (Scheme 4). Compound 9, endowed with a leaving group at the anomeric position and a free hydroxyl group at the 4'- position, and having a protecting group pattern encoding the negative charge distribution of the regular region of heparin was then submitted to autocondensation conditions.
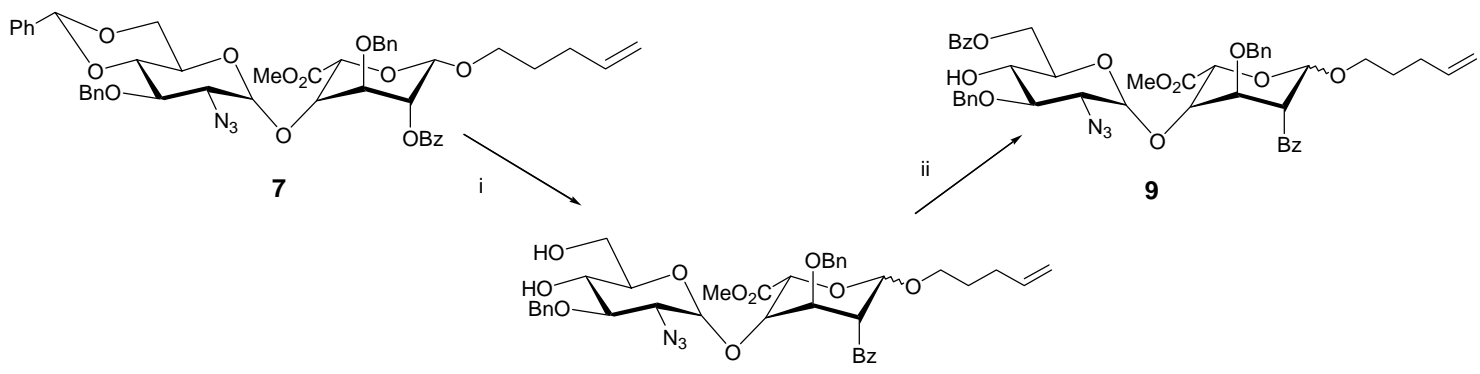

8

Scheme 4. (i) EtSH, $\mathrm{BF}_{3} \mathrm{OEt}_{2}, \mathrm{CH}_{2} \mathrm{Cl}_{2}, 72 \%$; (ii) $\mathrm{BzCN}_{2} \mathrm{Et}_{3} \mathrm{~N}, \mathrm{CH}_{2} \mathrm{Cl}_{2}, 92 \%$.

It was expected that the reaction of 9 with a promoter (equimolecular NIS and catalytic $\mathrm{Et}_{3} \mathrm{SiOTf}^{19}$ ) would generate a glycosyl carbonium ion ${ }^{13 \mathrm{~b}}$ that may react with any nucleophile present in the reaction mixture. Under strictly anhydrous conditions, the carbonium ion would 
react with the 4'-OH group of a second disaccharide building block to give a tetrasaccharide species that itself carries donor-acceptor functionalities. This tetrasaccharide species may give rise to a hexasaccharide or an octasaccharide depending on the concentration of each species, the nucleophilicity of the acceptor $\mathrm{OH}$ group, and the concentration of the promoter.

Compound 9 was dissolved in dichloromethane under strictly anhydrous conditions and treated with solid NIS and a catalytic amount of $\mathrm{Et}_{3} \mathrm{SiOTf}^{19}$ at $0^{\circ} \mathrm{C}$. The reaction was monitored by TLC. When no further evolution was observed the reaction mixture was acetylated by treatment with acetic anhydride and pyridine in the presence of DMAP. ${ }^{20}$ It was then submitted to extensive TLC fractionation and compounds 10-13 could be isolated and characterized (Scheme 5). The presence of the $N$-succinyl derivatives $\mathbf{1 0}$ and $\mathbf{1 1}$ indicated that under the reaction conditions the succinimide nucleophile competes strongly with the 4'-OH group for the NIS-Et ${ }_{3}$ SiOTf- generated glycosyl carbonium ion, and this leads to a rapid end of the chain reaction. The formation of $\mathrm{N}$-succinyl glycosides has been reported as a side reaction in the glycosylation of glycosyl acceptors of low reactivity with thioglycosides. ${ }^{21}$ The fact that $N$ succinyl glycosides are formed at the disaccharide level (compound 11), and the absence of higher oligomers in the reaction mixture, are clear indications of the limited efficiency of this offset for autocondensation reactions.

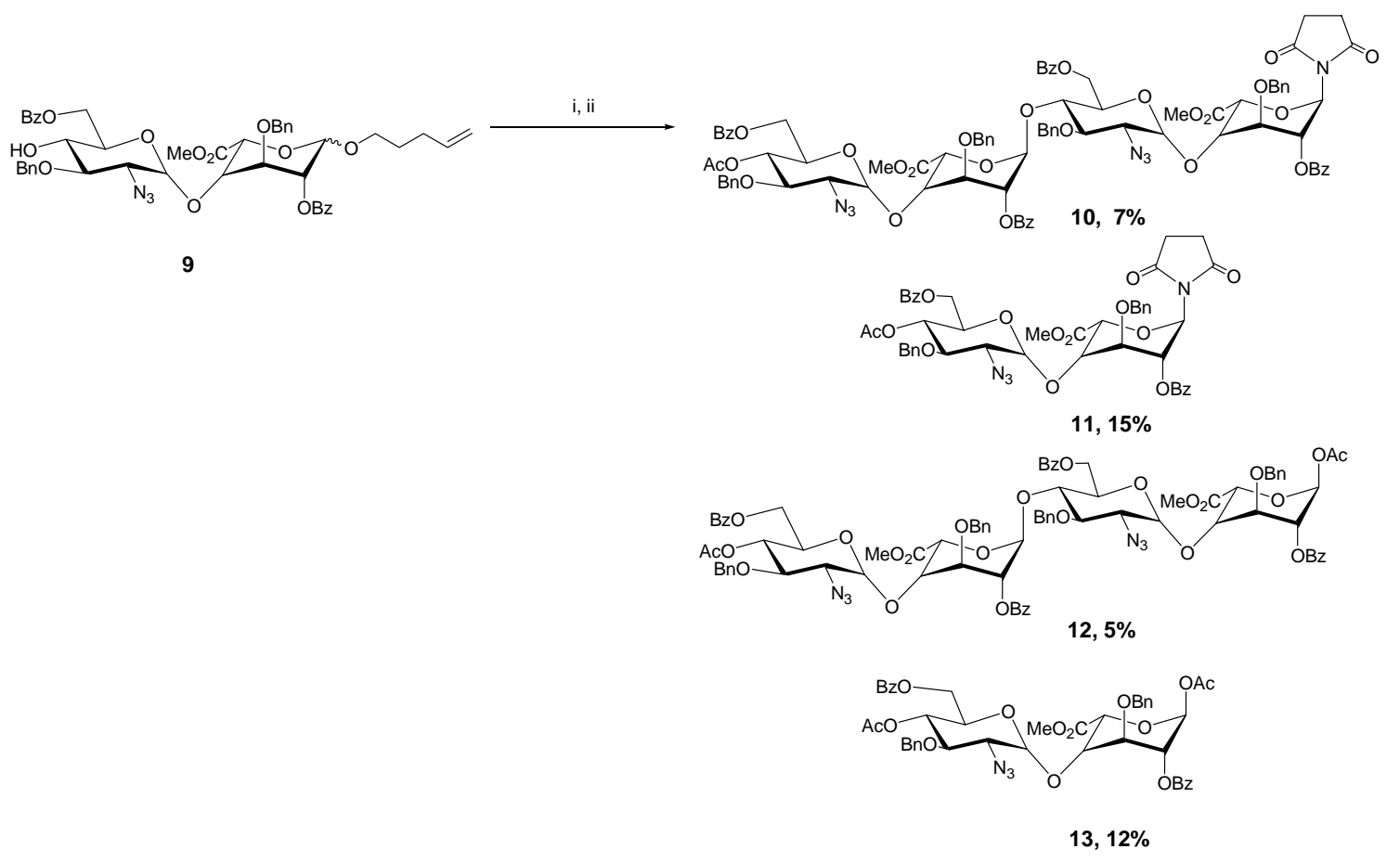

Scheme 5. (i) $\mathrm{Et}_{3} \mathrm{SiOTf}$, NIS, $\mathrm{CH}_{2} \mathrm{Cl}_{2}$; (ii) $\mathrm{Ac}_{2} \mathrm{O}$, pyridine.

When the reaction was carried out under TMSOTf catalysis with 1.1 eq. of NIS in dichloromethane, a fraction containing tetra- and hexa-saccharide was formed in $11 \%$ yield 
(calculated from (NMR). However, higher oligomers from subsequent glycosylation cycles could never be observed.

\section{Conclusions}

From this exploratory study it can be concluded that the glucosamine $\alpha 1 \rightarrow 4$ L-iduronate building block is not a suitable candidate for self-condensation, because it has low reactivity both as a glycosyl donor and as a glycosyl acceptor. In fact, uronic acids had a poor performance under the classical tritylcyanoethylidene polycondensation conditions. ${ }^{22}$ The use of a trichloroacetimidate function for anomeric activation seems to be precluded since the presence of a free 4'-OH in the disaccharide building block leads to intramolecular transesterification of the methyl ester with the free anomeric hydroxyl group during the attempted selective installation of the trichloroacetimidate group in the anomeric position. The use of more stable leaving groups, such as the n-pentenyl group, for anomeric activation allows protecting-group manipulation, but suffers from the fact that equimolecular amounts of promoter are required. Being a nucleophile itself, the promoter competes with the acceptor alcohol in the polycondensation process, which results in fast chain-reaction termination and a low yield and degree of polymerization. Since the preparation of the starting disaccharide building block involves a laborious process, the synthesis of homogeneous heparin oligosaccharide fragments by self-condensation does not seem to compete with the sequential assembly of disaccharide building blocks using classical deprotection-glycosylation strategies.

\section{Experimental Section}

General Procedures. Tetrahydrofuran, toluene, 1,4-dioxane and diethyl ether were distilled from sodium under nitrogen prior to use. Dichloromethane, and acetonitrile were distilled from calcium hydride and used immediately after distillation. DMF, pyridine and methanol were commercial high-grade solvents (SDS, Prolabo, France) and stored under Argon over molecular sieves (4A). All reagents were purchased from Aldrich, Fluka, Lancaster, Panreac, Acros, or SDS. All reactions were carried out under argon in pre-dried glassware, unless otherwise stated. Analytical thin layer chromatography (TLC) was performed on silica gel $60 \mathrm{~F}_{254}$ pre-coated on aluminum plates (Merck, Darmstadt) and the compounds detected by staining with phosphomolybdic acid/EtOH or with anisaldehyde solution [anisaldehyde $(25 \mathrm{ml})$ with sulfuric acid $(25 \mathrm{ml})$, ethanol $(450 \mathrm{ml})$ and acetic acid $(1 \mathrm{ml})]$ with detection by heating above $200^{\circ} \mathrm{C}$. Flash-column chromatography was carried out on silica gel $60(0.2-0.5 \mathrm{~mm}, 0.2-0.063 \mathrm{~mm}$ or 0.040-0.015 mm; Merck, Darmstadt) under a pressure of 0.3-0.8 bar. Preparative TLC purifications were carried out on Merck 20x20 cm silica-gel $60 \mathrm{~F}_{254}$-plates. Gel-permeation chromatography used Sephadex LH-20 and G-25 gels (medium and fine) from Pharmacia, on 
Pharmacia columns equipped with a fraction collector. Optical rotations were determined with a Perkin-Elmer 341 polarimeter at $589 \mathrm{~nm}$ and r.t. ${ }^{1} \mathrm{H}$ - and ${ }^{13} \mathrm{C}-\mathrm{NMR}$, as all two-dimensional spectra were acquired on Bruker DPX-300, DRX-400 and DRX-500 spectrometers, and chemical shifts are given in ppm $(\delta)$ relative to tetramethylsilane as internal reference or relative to $\mathrm{D}_{2} \mathrm{O}$. FAB MS were measured by the Mass Spectrometry Service, Facultad de Química, Seville, with a Kratos MS-80 RFA spectrometer, MALDI-TOF-MS were recorded on a Hewlett Packard 2030A system, usually with 2,5-dihydroxybenzoic acid (DHB-matrix). Elemental analysis was performed on a Leco CHNS-932 apparatus, after drying the sample over phosphorus pentoxide.

2-Azido-6-O-benzoyl-3-O-benzyl-2-deoxy-D-glucopyranosyl- $\alpha-(1 \rightarrow 4)$-methyl (2-O-pivaloyl-

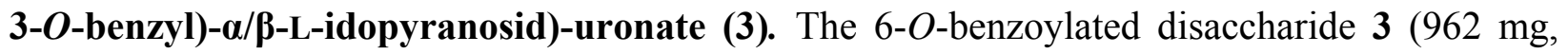
$1.06 \mathrm{mmol})$ was dissolved in THF $(30 \mathrm{ml})$ and the solution split in two portions and stirred under argon in PE-flasks. After cooling to $-20^{\circ} \mathrm{C}$, excess $\mathrm{HF}$ pyr $(2 \mathrm{ml} /$ for each charge $)$ was added via syringe and the reaction mixtures stirred at $4{ }^{\circ} \mathrm{C}$ for two days. $\mathrm{Et}_{2} \mathrm{O}(30 \mathrm{ml}$ each) was added for dilution and the crude reaction mixture neutralized with sat. $\mathrm{NaHCO}_{3}$-sol. and washed with brine. Concentration under reduced pressure followed by chromatography (ethyl acetate/hexane 2:1) of the crude product afforded $3(910 \mathrm{mg}, 1.0 \mathrm{mmol}, 94 \%)$ as a colorless foam. TLC (hexane/ethyl acetate 2:1) $\mathrm{R}_{\mathrm{f}} 0.24,[\alpha]^{\mathrm{rt}} \mathrm{D}+39.68^{\circ}\left(\right.$ c $\left.0.93, \mathrm{CHCl}_{3}\right) ;{ }^{1} \mathrm{H}-\mathrm{NMR}\left(500 \mathrm{MHz}, \mathrm{CDCl}_{3}\right)$ data for $\alpha$-anomer $\delta 8.01-7.99(\mathrm{~d}, J=7.1 \mathrm{~Hz}, 1 \mathrm{H}$; Bz), 7.71-7.26 (m, 13H; Bz, Bn), 5.38-5.35 (m, 1H; $\alpha-\mathrm{H}-1), 5.03$ (d, J $\left.J_{1,2}=3.6 \mathrm{~Hz}, 1 \mathrm{H}, \mathrm{H}-1^{\prime}\right), 4.88-4.75$ (m, 7H; H-2, H-6a', H-5, CH${ }_{2} \mathrm{Ph}$ ), 4.41-4.38 (m, 1H; H-6b'), 4.15-4.12 (m, 1H, H-4), 4.11-4.07 (m, 1H, H-3), 3.95-3.93 (m, 1H, H-5'), 3.79 (s, 3H; COOMe), 3.73-3.71 (m, 1H, OH-1'), 3.53-3.50 (m, 1H, H-4'), 3.21 (dd, $J=$ $3.6 \mathrm{~Hz}, J=10.2 \mathrm{~Hz}, 1 \mathrm{H}, \mathrm{H}-2^{\prime}$ ), 3.08 (bs, 1H, OH-4'), 1.23 (s, 9H, t-Bu). FAB-MS m/z 785 $\left(\mathrm{M}+\mathrm{Na}^{+}\right), \mathrm{C}_{39} \mathrm{H}_{45} \mathrm{~N}_{3} \mathrm{O}_{13}$ (763.79). Anal. Calcd. C, 61.33; H, 5.94; N, 5.50. Found C, 61.29; H, $6.03 ; \mathrm{N}, 5.63$.

\section{2-Azido-3-O-benzyl-4,6-di-O-benzylidene-2-deoxy-D-glucopyranosyl- $\alpha$-(1 $\rightarrow 4)-3-O$-benzyl-} $\boldsymbol{\alpha}$-L-idopyranosid)-6,1-lactone (4). A solution of $\mathbf{3}$ (70 $\mathrm{mg}, 0.091 \mathrm{mmol}$ ) in $\mathrm{CH}_{2} \mathrm{Cl}_{2}$ (2 ml) under inert gas was cooled to $0^{\circ} \mathrm{C}$ and after addition of trichloroacetonitrile $(11 \mu 1,0.109 \mathrm{mmol})$ was treated with freshly activated $\mathrm{K}_{2} \mathrm{CO}_{3}(14 \mathrm{mg}, 0.1 \mathrm{mmol})$. The mixture was stirred overnight at r.t., diluted with $\mathrm{CH}_{2} \mathrm{Cl}_{2}(2 \mathrm{ml})$, filtered through a pad of Celite and concentrated in vacuo. Compound 4 (0.058 mmol, $42 \mathrm{mg}, 64 \%)$ was isolated as the major product by column chromatography on silica gel (hexane/ethyl acetate 8:1). TLC (toluene/ethyl acetate 6:1) $\mathrm{R}_{\mathrm{f}}$ 0.46; $[\alpha]^{\mathrm{rt}}{ }_{\mathrm{D}}+50.68^{\circ}\left(\mathrm{c} 1.33, \mathrm{CHCl}_{3}\right) ;{ }^{1} \mathrm{H}-\mathrm{NMR}\left(500 \mathrm{MHz}, \mathrm{CDCl}_{3}\right) \delta 7.99$ (d, J = $\left.7.6 \mathrm{~Hz}, 2 \mathrm{H} ; \mathrm{Bz}\right)$, 7.57-7.24 (m, 13H; Ph), 5.82 (d, $\left.J_{1,2}=2.0 \mathrm{~Hz}, 1 \mathrm{H} ; \mathrm{H}-1\right), 5.12\left(\mathrm{~d}, J_{1^{\prime}, 2^{\prime}}=3.8 \mathrm{~Hz}, 1 \mathrm{H} ; \mathrm{H}-1^{\prime}\right), 5.00$ $\left(\mathrm{d}, J_{\text {gem }}=10.6 \mathrm{~Hz}, 1 \mathrm{H}, \mathrm{CH}_{2} \mathrm{Ph}\right), 4.93\left(\mathrm{dd}, J_{2,1}=1.8 \mathrm{~Hz}, J_{2,3}=7.7 \mathrm{~Hz}, 1 \mathrm{H} ; \mathrm{H}-2\right), 4.89(\mathrm{~s}, 2 \mathrm{H}$; $\left.\mathrm{CH}_{2} \mathrm{Ph}\right), 4.80\left(\mathrm{dd}, J_{5^{\prime}, 6^{\prime}}=4.1 \mathrm{~Hz}, J_{6^{\prime} \mathrm{a}, 6^{\prime} \mathrm{b}}=12.4 \mathrm{~Hz}, 1 \mathrm{H} ; \mathrm{H}-6^{\prime} \mathrm{a}\right), 4.71$ (d, $\left.J_{5,4}=3.75 \mathrm{~Hz}, 1 \mathrm{H} ; \mathrm{H}-5\right)$, $4.65\left(\mathrm{~d}, J_{\mathrm{gem}} 10.6 \mathrm{~Hz}, 1 \mathrm{H} ; \mathrm{CH}_{2} \mathrm{Ph}\right), 4.49\left(\mathrm{dd}, J_{5^{\prime}, 6^{\prime}}=1.8 \mathrm{~Hz}, J_{6^{\prime} \mathrm{a}, 6^{\prime} \mathrm{b}}=12.4 \mathrm{~Hz} ; \mathrm{H}-6^{\prime} \mathrm{b}\right), 4.36-4.34$ (m, 1H; H-5'), 3.96-3.91 (m, 2H; H-3, H-4), 3.81 (t, $\left.J_{3^{\prime}, 4^{\prime}}=J_{2^{\prime}, 3^{\prime}}=9.2 \mathrm{~Hz} ; 1 \mathrm{H}, \mathrm{H}-3^{\prime}\right), 3.60-3.56$ $\left(\mathrm{m}, 1 \mathrm{H} ; \mathrm{H}-4^{\prime}\right), 3.49\left(\mathrm{dd}, J_{2^{\prime}, 1^{\prime}}=3.8 \mathrm{~Hz}, J_{2^{\prime}, 3^{\prime}}=10.2 \mathrm{~Hz} ; 1 \mathrm{H}, \mathrm{H}-2^{\prime}\right), 2.95\left(\mathrm{~d}, J_{4^{\prime \prime}}, \mathrm{OH}-4^{\prime}=2.9 \mathrm{~Hz}\right.$, 
1H; OH-4'), 1.22 (s, 9H; t-Bu). FAB-MS m/z $754\left(\mathrm{M}+\mathrm{Na}^{+}\right), 772\left(\mathrm{M}+\mathrm{K}^{+}\right), \mathrm{C}_{38} \mathrm{H}_{41} \mathrm{~N}_{3} \mathrm{O}_{12}(731.75)$. Anal. Calcd. C, 62.37; H, 5.65; N, 5.74; Found C, 62.40; H, 5.71; N, 5.74.

$O$-(Methyl 4-O-(2-azido-3-O-benzyl-6-O-benzoyl-2-deoxy-4-O-trichloracetamido- $\alpha$-D-glucopyranosyl)-3-O-benzyl-2-O-pivaloyl- $\alpha / \beta-\mathrm{L}$-idopyranosyluronate) trichloro- acetimidate (5). To a solution of $3(100 \mathrm{mg}, 0.11 \mathrm{mmol})$ in $\mathrm{CH}_{2} \mathrm{Cl}_{2}(2 \mathrm{ml})$ was added trichloracetonitrile $(165 \mu \mathrm{l}$, $1.65 \mathrm{mmol}$ ) and one drop of DBU at r.t. The reaction mixture was stirred overnight, diluted with $\mathrm{CH}_{2} \mathrm{Cl}_{2}(3 \mathrm{ml})$ and the solvents removed under reduced pressure. The resulting oil was purified on a silica-gel column (toluene/ethyl acetate, 40:1) to afford the bis-trichloroacetimidate 5 (41 mg, $0.39 \mathrm{mmol})$ and lactone $4(38 \mathrm{mg}, 0.052 \mathrm{mmol})$. Data for trichloroacetimidate 5: TLC (hexane/ethyl acetate 3:1) $\mathrm{R}_{\mathrm{f}} 0.47 ;[\alpha]^{\mathrm{rt}}+30.42^{\circ}\left(c 1.69, \mathrm{CHCl}_{3}\right),{ }^{1} \mathrm{H}-\mathrm{NMR}\left(500 \mathrm{MHz}, \mathrm{CDCl}_{3}\right) \delta$ 8.70 (s, 1H; NH), 8.62 (s, 1H; NH), 8.03 (d, $J=7.3 \mathrm{~Hz}, 2 \mathrm{H} ; \mathrm{Bz}), 8.00-726$ (m, 13H; Ph), 6.41 $(\mathrm{s}, 1 \mathrm{H} ; \mathrm{H}-1), 5.65$ (t, $\left.J_{4,3}=J_{4,5}=9.7 \mathrm{~Hz}, 1 \mathrm{H} ; \mathrm{H}-4^{\prime}\right), 5.18\left(\mathrm{t}, J_{1,2}=J_{2,3}=2.6 \mathrm{~Hz}, 1 \mathrm{H} ; \mathrm{H}-2\right), 5.03$ $\left(\mathrm{d}, J_{1^{\prime}, 2^{\prime}}=3.6 \mathrm{~Hz}, 1 \mathrm{H}, \mathrm{H}-1^{\prime}\right), 4.94\left(\mathrm{~d}, J_{5,4}=2.6 \mathrm{~Hz}, 1 \mathrm{H} ; \mathrm{H}-5\right), 4.82\left(\mathrm{~d}, J_{\text {gem }}=11.4 \mathrm{~Hz}, 1 \mathrm{H}\right.$; $\left.\mathrm{CH}_{2} \mathrm{Ph}\right), 4.77\left(\mathrm{~d}, J_{\text {gem }}=10.1 \mathrm{~Hz}, 1 \mathrm{H} ; \mathrm{CH}_{2} \mathrm{Ph}\right), 4.72\left(\mathrm{~d}, J_{\text {gem }}=11.4 \mathrm{~Hz}, 1 \mathrm{H} ; \mathrm{CH}_{2} \mathrm{Ph}\right), 4.62(\mathrm{dd}$, $\left.J_{6^{\prime} \mathrm{a}, 5^{\prime}}=1.5 \mathrm{~Hz}, J_{6^{\prime} \mathrm{a}, 6^{\prime} \mathrm{b}}=12.5 \mathrm{~Hz}, 1 \mathrm{H} ; \mathrm{H}-6^{\prime} \mathrm{a}\right), 4.34-4.28\left(\mathrm{~m}, 2 \mathrm{H} ; \mathrm{H}-5^{\prime}, \mathrm{H}-6^{\prime} \mathrm{b}\right), 4.25\left(\mathrm{t}, J_{4,5}=J_{4,3}\right.$ $=3.6 \mathrm{~Hz}, 1 \mathrm{H} ; \mathrm{H}-4), 4.09\left(\mathrm{t}, J_{3^{\prime}, 4^{\prime}}=J_{3^{\prime}, 2^{\prime}}=9.7 \mathrm{~Hz}, 1 \mathrm{H} ; \mathrm{H}-3^{\prime}\right), 4.04\left(\mathrm{t}, J_{3,4}=J_{3,2}=3.4 \mathrm{~Hz}, 1 \mathrm{H}\right.$; H3), $3.81\left(\mathrm{~s}, 3 \mathrm{H} ; \mathrm{COOCH}_{3}\right), 3.51\left(\mathrm{dd}, J_{2^{\prime}, 1^{\prime}}=3.6 \mathrm{~Hz}, J_{2^{\prime}, 3^{\prime}}=10.2 \mathrm{~Hz}, 1 \mathrm{H}, \mathrm{H}-2^{\prime}\right), 1.28(\mathrm{~s}, 9 \mathrm{H}$; $\left.\mathrm{C}\left(\mathrm{CH}_{3}\right)_{3}\right),{ }^{13} \mathrm{C}-\mathrm{NMR}\left(125 \mathrm{MHz}, \mathrm{CDCl}_{3}\right) \delta 177.44,166.07,161.45,137.09,133.16,133.09$, $128.85,129.79,129.71,128.61,128.58,128.49,128.45,128.43,128.30,128.21,128.12,127.98$, $127.81,127.78,127.73,127.67,99.15,85.61,93.57,78.10,77.96,75.23,75.06,74.49,74.11$, 73.76, 72.76, 61.99, 52.62, 38.97, 38.94, 29.71, 27.41, 27.30, 27.26, 27.02. FAB-MS m/z 931 $\left(\mathrm{M}-1 \mathrm{TCA}+\mathrm{Na}^{+}\right), 786\left(\mathrm{M}-2 \mathrm{TCA}+\mathrm{Na}^{+}\right), \mathrm{C}_{43} \mathrm{H}_{45} \mathrm{Cl}_{6} \mathrm{~N}_{5} \mathrm{O}_{13}(1052.56)$.

2-Azido-3-O-benzyl-4,6-benzylidene-2-deoxy-D-glucopyranosyl- $\alpha-(1 \rightarrow 4)$ methyl (n-pentenyl 2-O-benzoyl-3-O-benzyl- $\boldsymbol{\alpha}$-L-idopyranosid)-uronate (7). A solution of $\mathbf{6}^{3 \mathrm{c}}(59 \mathrm{mg}$, $0.284 \mathrm{mmol})$ and 4-penten-1-ol in $\mathrm{CH}_{2} \mathrm{Cl}_{2}(2 \mathrm{ml})$ was cooled to $0^{\circ} \mathrm{C}$ and $50 \mu \mathrm{l}$ of a freshly prepared TMSOTf- solution $(0.03 M)$ added via syringe. The mixture was allowed to warm to r.t. during $30 \mathrm{~min}$, diluted with $\mathrm{CH}_{2} \mathrm{Cl}_{2}(5 \mathrm{ml})$ and neutralized with sat. $\mathrm{NaHCO}_{3}$ solution $(4 \mathrm{ml})$. The separated organic phase was washed with brine, dried over $\mathrm{MgSO}_{4}$ and concentrated in vacuo. The residue was eluted from a column of silica gel $(4: 1 \rightarrow 2: 1)$ hexane/ethyl acetate) to afford 7 (112 mg, $0.134 \mathrm{mmol}, 47 \%$ ), the corresponding trichloroacetamide (47 $\mathrm{mg}, 0.051 \mathrm{mmol}$, $18 \%$ ), and the hydrolyzed 1-OH-free compound (76 mg, $0.1 \mathrm{mmol}, 35 \%)$. Data for 7: TLC (8:1 toluene/ethyl acetate) $\mathrm{R}_{\mathrm{f}} 0.62 ;{ }^{1} \mathrm{H}-\mathrm{NMR}\left(500 \mathrm{MHz}, \mathrm{CDCl}_{3}\right) \delta 8.17-8.14(\mathrm{~m}, 2 \mathrm{H} ; \mathrm{Bz}) 7.52-7.13$ (m, 18H, Ph), 5.85-5.73 (m, 1H; $\left.\mathrm{CH}=\mathrm{CH}_{2}\right), 5.53(\mathrm{~s}, 1 \mathrm{H} ; \mathrm{PhCH}), 5.22$ (bs, 1H; H-1), 5.17 (bs, $1 \mathrm{H}$; H-2), 5.02-4.95 (m, 3H; $\mathrm{CH}=\mathrm{CH}_{2}, \mathrm{CH}_{2} \mathrm{Ph}$ ), 4.89 (bs, 1H; H-5), 4.78-4.74 (m, 2H; H-1'), 4.43-4.35 (m, 2H, CH ${ }_{2} \mathrm{Ph}, \mathrm{H}-6$ 'a), 4.17 (bs, 1H; H-3), 4.12 (bs, 1H; H-4), 4.08-4.00 (m, 1H, H6'b), 3.94 (d, $J=10.7 \mathrm{~Hz}, 1 \mathrm{H} ; \mathrm{CH}_{2} \mathrm{Ph}$ ), 3.87-3.79 (m, 4H, $\mathrm{COOCH}_{3}, \mathrm{CH}_{2} \mathrm{Ph}$ ), 3.68-3.53 (m, 4H, $\mathrm{CH}_{2} \mathrm{Ph}, \mathrm{H}-3^{\prime}, \mathrm{H}-4^{\prime}, \mathrm{H}-5^{\prime}$ ), 3.21 (dd, $\left.J_{2^{\prime}, 1^{\prime}}=3.5 \mathrm{~Hz}, J_{2^{\prime}, 3^{\prime}}=9.3 \mathrm{~Hz}, 1 \mathrm{H}, \mathrm{H}-2^{\prime}\right)$ 2.18-2.11 (m, 2H; $\mathrm{CH}_{2}$ (n-Pent.)), 1.77-1.72 (m, 2H; $\mathrm{CH}_{2}$ (n-Pent.)), 1.26 (m, 2H; $\mathrm{CH}_{2}$ (n-Pent.)), ${ }^{13} \mathrm{C}-\mathrm{NMR}(125$ $\left.\mathrm{MHz}, \mathrm{CDCl}_{3}\right) \delta 170.10,166.00,138.40,138.19,138.03,137.91,133.66,130.36,130.06,129.45$, $129.15,128.78,128.71,128.66,128.35,128.34,128.29,128.16,126.53,126.51,115.40,101.82$, 
100.22, 99.63, 82.80, 76.96, 76.42, 75.13, 73.55, 72.66, 68.94, 68.43, 68.00, 67.51, 63.86, 63.54, 52.68, 30.64, 28.96. Anal. Calcd. for $\mathrm{C}_{46} \mathrm{H}_{49} \mathrm{~N}_{3} \mathrm{O}_{2} \mathrm{C}, 66.10 ; \mathrm{H}, 5.91 ; \mathrm{N}, 5.03$; Found C, 66.35; H, $6.08 ; \mathrm{N}, 5.14$.

2-Azido-3-O-benzyl-2-deoxy-D-glucopyranosyl- $\alpha-(1 \rightarrow 4)$ - methyl (n-pentenyl 2-O-benzoyl-3O-benzyl-a-L-idopyranosid)-uronate (8). Compound 7 (148 $\mathrm{mg}, 0.177 \mathrm{mmol})$ was dissolved in $\mathrm{CH}_{2} \mathrm{Cl}_{2}(3 \mathrm{ml})$, cooled to $0^{\circ} \mathrm{C}$, and $\mathrm{EtSH}(200 \mu \mathrm{l}, 2.66 \mathrm{mmol})$ added under stirring. Freshly prepared $\mathrm{BF}_{3} \mathrm{OEt}_{2}$-sol. $(50 \mu \mathrm{l}, 0.02 \mathrm{M})$ was injected via syringe and the reaction mixture allowed to warm up to r.t. After stirring for $3 \mathrm{~h}$, TLC- analysis indicated complete consumption of the starting material. The acid was neutralized by addition of excess $\mathrm{Et}_{3} \mathrm{~N}(500 \mu \mathrm{l})$ and the solvents removed under reduced pressure. The residue was purified by column chromatography $(1: 1$ hexane/ethyl acetate) affording 8 (92 $\mathrm{mg}, 0.13 \mathrm{mmol}, 72 \%)$ as a colorless oil.

TLC (2:1 toluene/ethyl acetate) $\mathrm{R}_{\mathrm{f}} 0.46 ;{ }^{1} \mathrm{H}-\mathrm{NMR}\left(300 \mathrm{MHz}, \mathrm{CDCl}_{3}\right) \delta 8.18-8.15$ (m, 2H; Bz), 7.55-7.14 (m, 13H, Ph), 5.86-5.72 (m, 1H; $\left.\mathrm{CH}=\mathrm{CH}_{2}, \mathrm{CH}_{2} \mathrm{Ph}\right), 5.21$ (bs, $\left.1 \mathrm{H} ; \mathrm{H}-1\right), 5.15$ (bs, $1 \mathrm{H}$; $\mathrm{H}-2$ ), 5.01-4.93 (m, 3H; $\left.\mathrm{CH}=\mathrm{CH}_{2}, \mathrm{CH}_{2} \mathrm{Ph}\right), 4.88$ (d, $\left.J_{5,4}=1.96 \mathrm{~Hz}, 1 \mathrm{H} ; \mathrm{H}-5\right), 4.76-4.74$ (m, 2H; $\left.\mathrm{CH}_{2} \mathrm{Ph}\right), 4.22\left(\mathrm{~d}, J_{\text {gem }}=11.0 \mathrm{~Hz} ; 1 \mathrm{H}, \mathrm{CH}_{2} \mathrm{Ph}\right), 4.17-4.08\left(\mathrm{~m}, 3 \mathrm{H} ; \mathrm{H}-3, \mathrm{H}-4, \mathrm{CH}_{2} \mathrm{Ph}\right), 3.86-3.76$

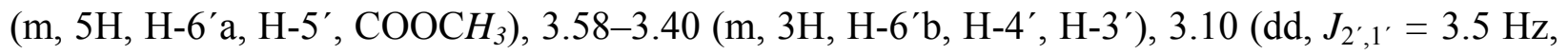
$\left.J_{2^{\prime}, 3^{\prime}}=9.6 \mathrm{~Hz}, 1 \mathrm{H} ; 1 \mathrm{H}, \mathrm{H}-2^{\prime}\right), 2.92\left(\mathrm{~d}, J_{\mathrm{OH}, 4^{\prime}}=3.0 \mathrm{~Hz}, 1 \mathrm{H} ; \mathrm{OH}-4^{\prime}\right), 2.31$ (bs, 1H; OH-6'), $2.17-$ 2.10 (m, 2H, $\mathrm{CH}_{2}$ (n-pent.)), $1.75-1.68$ (m, 4H; $\mathrm{CH}_{2}$ (n-pent.)), ${ }^{13} \mathrm{C}-\mathrm{NMR}\left(75 \mathrm{MHz}, \mathrm{CDCl}_{3}\right) \delta$ 202.76, 179.71, 170.51, 166.03, 138.38, 138.20, 138.00, 133.79, 130.46, 130.10, 129.40, 129.11, $128.94,128.78,128.59,128.38,128.31,128.21,120.35,115.40,99.66,99.51,80.31,75.88$, 75.10, 73.37, 73.00, 72.69, 71.54, 68.44, 68.30, 67.52, 63.57, 62.79, 52.86, 30.61, 28.94. Anal. Calcd. for $\mathrm{C}_{39} \mathrm{H}_{45} \mathrm{~N}_{3} \mathrm{O}_{11}$ C, 64.01; H, 6.20; N, 5.74; Found C, 64.12; H, 6.18; N, 5.77.

2-Azido-6-O-benzoyl-3-O-benzyl-2-deoxy-D-glucopyranosyl- $\alpha-(1 \rightarrow 4)$ methyl (n-pentenyl 2$\boldsymbol{O}$-benzoyl-3-O-benzyl-a-L-idopyranosid)-uronate (9). Compound 8 (133 $\mathrm{mg}, 0.178 \mathrm{mmol}$ ) in $\mathrm{CH}_{3} \mathrm{CN}(3 \mathrm{ml})$ was cooled to $-40^{\circ} \mathrm{C}$ and treated with $200 \mu \mathrm{l}$ of a freshly prepared $\mathrm{BzCN}$ solution $(0.9 \mathrm{M}, 0.18 \mathrm{mmol})$ in $\mathrm{CH}_{3} \mathrm{CN}$. Upon addition of cat. $\mathrm{Et}_{3} \mathrm{~N}(10 \mu \mathrm{l})$ stirring was continued at $40^{\circ} \mathrm{C}$ for $1.5 \mathrm{~h}$. To drive the reaction to completion, addition of $20 \mu \mathrm{l}$ of $\mathrm{BzCN}$-sol. (0.9M, $0.018 \mathrm{mmol}$ ) and stirring for $30 \mathrm{~min}$ was necessary. The reaction was quenched with $\mathrm{MeOH}$ $(1 \mathrm{ml})$, the solvents removed under reduced pressure, and the residue subjected to column chromatography (8:1 toluene/ethyl acetate) to afford 9 (136 mg, $0.16 \mathrm{mmol}, 92 \%)$ as a colorless oil.

TLC (4:1 toluene/ethyl acetate) $\mathrm{R}_{\mathrm{f}} 0.71 ;{ }^{1} \mathrm{H}-\mathrm{NMR}\left(300 \mathrm{MHz}, \mathrm{CDCl}_{3}\right) \delta 8.15(\mathrm{~d}, J=7.3 \mathrm{~Hz}, 2 \mathrm{H}$; $\mathrm{Bz}), 8.00(\mathrm{~d}, J=8.1 \mathrm{~Hz}, 2 \mathrm{H} ; \mathrm{Bz}), 7.99-7.17(\mathrm{~m}, 16 \mathrm{H} ; \mathrm{Ph}), 5.80-5.73\left(\mathrm{~m}, 1 \mathrm{H} ; \mathrm{CH}=\mathrm{CH}_{2}\right), 5.18$ (bs, 1H; H-2), 5.16 (bs, 1H; H-1), 4.99-4.84 (m, 4H; $\mathrm{CH}=\mathrm{CH}_{2}, \mathrm{CH}_{2} \mathrm{Ph}, \mathrm{H}-5, \mathrm{H}-6$ 'a), 4.81 (d, $\left.J_{1^{\prime}, 2^{\prime}}=3.4 \mathrm{~Hz}, 1 \mathrm{H} ; \mathrm{H}^{\prime} 1^{\prime}\right), 4.73\left(\mathrm{~d}, J_{\mathrm{gem}}=11.6 \mathrm{~Hz}, 1 \mathrm{H} ; \mathrm{CH}_{2} \mathrm{Ph}\right), 4.43\left(\mathrm{dd}, J_{5^{\prime}, 6^{\prime} \mathrm{b}}=1.9 \mathrm{~Hz}, J_{6^{\prime} \mathrm{a}, 6^{\prime} \mathrm{b}}\right.$ $=12.4 \mathrm{~Hz}, 1 \mathrm{H}, \mathrm{H}-6$ 'b), 4.31 (d, $\left.J_{\text {gem }}=10.8 \mathrm{~Hz}, 1 \mathrm{H} ; \mathrm{CH}_{2} \mathrm{Ph}\right), 4.06-3.96$ (m, 2H; H-3, H-4), 3.813.80 (m, 4H; $\mathrm{COOCH}_{3}, \mathrm{CH} 2$ (n-pent.)), 3.55-3.42 (m, 3H; H-3', $\mathrm{CH}_{2}$ (n-pent.), H-4'), 3.12 (dd, $\left.J_{2^{\prime}, 1^{\prime}}=3.4 \mathrm{~Hz}, J_{2^{\prime}, 3^{\prime}}=9.9 \mathrm{~Hz}, 1 \mathrm{H} ; \mathrm{H}-2^{\prime}\right), 2.92\left(\mathrm{~d}, J_{\mathrm{OH}-4^{\prime}, 4^{\prime}}=3.8 \mathrm{~Hz}, 1 \mathrm{H} ; \mathrm{OH}-4^{\prime}\right), 2.13-2.09$ (m, 2H; $\mathrm{CH}_{2}$ (n-pent.)), 1.75-1.66 (m, 2H; $\mathrm{CH}_{2}$ (n-pent.)), ${ }^{13} \mathrm{C}-\mathrm{NMR}\left(125 \mathrm{MHz}, \mathrm{CDCl}_{3}\right) \delta 169.86$, $167.48,165.61,137.98,137.85,137.66,133.37,130.20,130.05,129.87,129.72,129.49,128.75$, 
$128.65,128.55,128.44,128.36,128.07,127.98,127.89,127.85,114.97,114.83,99.36,99.24$, $79.27,75.67,74.98,73.24,72.33,71.40,70.48,68.07,67.92,67.39,63.22,63.02,52.34,30.21$, 30.06, 28.60. FAB-MS m/z $874\left(\mathrm{M}+\mathrm{Na}^{+}\right), \mathrm{C}_{46} \mathrm{H}_{49} \mathrm{~N}_{3} \mathrm{O}_{12}$ (835.89). Anal. Calcd. C, 66.10; H, 5.91; N, 5.03; Found C, 66.22; H, 6.18; N, 5.12.

Autocondensation of 9. Compound $9(66.4 \mathrm{mg}, 0.026 \mathrm{mmol})$ was co-evaporated four times with freshly distilled toluene $(1.5 \mathrm{ml}$ each) and dried in high vacuum for $2 \mathrm{~h}$. The dried compound was then dissolved in $\mathrm{CH}_{2} \mathrm{Cl}_{2}(1 \mathrm{ml})$ and stirred for $30 \mathrm{~min}$ in the presence of activated molecular sieves ( $3 \AA \hat{A})$. After cooling to $0^{\circ} \mathrm{C}$, NIS $(19 \mathrm{mg}, 0.086 \mathrm{mmol})$ was added as a solid, followed by the slow addition of $50 \mu \mathrm{l}$ of a freshly prepared $\mathrm{Et}_{3} \mathrm{SiOTf}_{-s o l}(0.044 \mathrm{M}, 0.023 \mathrm{mmol})$. The reaction was stirred for $15 \mathrm{~min}$ at $0^{\circ} \mathrm{C}$ then warmed up to r.t. The evolution of the reaction was monitored by TLC at intervals of $5 \mathrm{~min}$. After $70 \mathrm{~min}$ the reaction was diluted with $\mathrm{CH}_{2} \mathrm{Cl}_{2}$ $(5 \mathrm{ml})$ and quenched by injection of sat. $\mathrm{NaHCO}_{3}$ solution $(2 \mathrm{ml})$. The separated organic phase was washed successively with sat. $\mathrm{Na}_{2} \mathrm{~S}_{2} \mathrm{O}_{3}$ solution and brine, dried over $\mathrm{MgSO}_{4}$ and concentrated in vacuo. The resulting residue was acetylated with acetic anhydride/pyridine (1:2, $1 \mathrm{ml}$ ) in the presence of a catalytic amount of DMAP for $2 \mathrm{~h}$ at $0^{\circ} \mathrm{C}$. After removal of the solvents by extraction with ethyl acetate/water, and co-evaporation with toluene to remove traces of pyridine, the crude residue was purified by column chromatography (toluene /ethyl acetate 3:1) to furnish a mixture of oligosaccharides which was further separated by preparative TLC (toluene /ethyl acetate 5:1) into three fractions (fr. 1, $18.8 \mathrm{mg}$; fr. 2, $7 \mathrm{mg}$; fr. 3, $47 \mathrm{mg}$ ) and analyzed by NMR spectroscopy. The following compounds were isolated.

Methyl (acetyl $O$-(4-O-actetyl-2-azido-6-O-benzoyl-3-O-benzyl-2-deoxy- $\alpha$-Dgluco-pyranosyl)-( $\rightarrow 4)-O$-methyl $\quad(2-O$-benzoyl-3-O-benzyl- $\alpha$-L-idopyranosyluronate)$(1 \rightarrow 4)-O$-(2-azido-6-O-benzoyl-3-O-benzyl-2-deoxy- $\alpha$-D-glucopyranosyl)-(1 $\rightarrow 4)-(2-O-$

benzoyl-3-O-benzyl-a-L-idopyranosyl) uronate (10). Compound 10 (9 mg, 0.0054 mmol, 7\%) was first isolated from the above reaction mixture. ${ }^{1} \mathrm{H}-\mathrm{NMR}\left(500 \mathrm{MHz}, \mathrm{CDCl}_{3}\right) \delta 8.07-7.89(\mathrm{~m}$, $8 \mathrm{H}$; ortho Bz), $7.60-7.00$ (m, 32H; Ph), 5.53 (d, $\left.J_{1,2}=3.2 \mathrm{~Hz}, 1 \mathrm{H} ; \mathrm{H}-1 \mathrm{a}\right), 5.34-5.29$ (m, $1 \mathrm{H}$; H1c), 5.16-5.12 (m, 2H, H-2a, H-4d), 4.98 (d, J3.6 = 3.6 Hz, H-1b), 4.90-4.84 (m, 3H; $\mathrm{CH}_{2} \mathrm{Ph}, \mathrm{H}-$ 1d), 4.75-4.72 (m, 2H, CH $2 \mathrm{Ph}), 4.70-4.63$ (m, 4H; H-5c, H-5a, $\left.\mathrm{CH}_{2} \mathrm{Ph}\right), 4.56$ (dd, $J_{\text {gem }}=12 \mathrm{~Hz}$, $2 \mathrm{H}, \mathrm{CH}_{2} \mathrm{Ph}$ ), 4.49-4.45 (m, 2H; H-6b, H-6d), 4.39 (bs, 1H, H-3a), 4.25 (m, 1H, H-4a), 4.18-3.99 (m, 5H, H-3c, H-4c, H-6b', H-5b, H-6d'), 3.91-3.84 (m, 2H, H-5d, H-4b), 3.66 (s, 3H; $\mathrm{COOCH}_{3}$ ), 3.61-3.55 (m, 2H; H-3d, H-3b), 3.46 (s, 3H, $\left.\mathrm{COOCH}_{3}\right), 3.33-3.27$ (m, 2H; H-2b, H2d), $1.94\left(\mathrm{~s}, 3 \mathrm{H} ; \mathrm{COCH}_{3}\right), 1.55\left(\mathrm{~s}, 3 \mathrm{H}, \mathrm{COCH}_{3}\right)$. FAB-MS m/z $1694\left(\mathrm{M}+\mathrm{Na}^{+}\right), \mathrm{C}_{88} \mathrm{H}_{85} \mathrm{~N}_{7} \mathrm{O}_{27}$ (1672.75).

4-O-Acetyl-2-azido-6-O-benzoyl-3-O-benzyl-2-deoxy-D-glucopyranosyl- $\alpha$-(1 $\rightarrow 4)$ - $O$-methyl ( $\mathrm{N}$-succinimido 2-O-benzoyl-3-O-benzyl-1-deoxy- $\alpha$-L-idopyranosid)-uronate (11). (8.2 $\mathrm{mg}$, $0.0094 \mathrm{mmol}, 12 \%) .{ }^{1} \mathrm{H}-\mathrm{NMR}\left(500 \mathrm{MHz}, \mathrm{CDCl}_{3}\right) \delta=8.02-7.93(\mathrm{~m}, 4 \mathrm{H} ; \mathrm{Bz}), 7.57-7.28(\mathrm{~m}$, $16 \mathrm{H}, \mathrm{Ph}), 6.17\left(\mathrm{~d}, J_{1,2}=9.4 \mathrm{~Hz}, 1 \mathrm{H} ; \mathrm{H}-1\right), 6.13\left(\mathrm{t}, J_{2,1}=J_{2,3}=8.3 \mathrm{~Hz}, 1 \mathrm{H} ; \mathrm{H}-2\right), 5.25\left(\mathrm{~d}, J_{1^{\prime}, 2^{\prime}}=\right.$ $\left.3.6 \mathrm{~Hz}, 1 \mathrm{H} ; \mathrm{H}^{\prime} 1^{\prime}\right), 5.16\left(\mathrm{t}, J_{4^{\prime}, 3^{\prime}}=J_{4^{\prime}, 5^{\prime}}=5.75 \mathrm{~Hz}, 1 \mathrm{H} ; \mathrm{H}-4^{\prime}\right), 4.87$ (d, $\left.J_{5,4}=5.75 \mathrm{~Hz}, 1 \mathrm{H} ; \mathrm{H}-5\right)$, $4.82\left(\mathrm{~d}, J_{\text {gem }}=10.7 \mathrm{~Hz}, 1 \mathrm{H}, \mathrm{CH}_{2} \mathrm{Ph}\right), 4.80\left(\mathrm{~d}, J_{\text {gem }} 10.7 \mathrm{~Hz}, 1 \mathrm{H} ; \mathrm{CH}_{2} \mathrm{Ph}\right), 4.69\left(\mathrm{~d}, J_{\text {gem }}=11 \mathrm{~Hz}\right.$, $\left.1 \mathrm{H} ; \mathrm{CH}_{2} \mathrm{Ph}\right), 4.63\left(\mathrm{~d}, J_{\mathrm{gem}} 11 \mathrm{~Hz}, 1 \mathrm{H} ; \mathrm{CH}_{2} \mathrm{Ph}\right), 4.46\left(\mathrm{dd}, J_{5^{\prime}, 6^{\prime} \mathrm{a}}=2.4 \mathrm{~Hz}, J_{6^{\prime} \mathrm{a}, 6^{\prime} \mathrm{b}}=12.3 \mathrm{~Hz}, 1 \mathrm{H}\right.$; H- 
6'a), 4.39-4.31 (m, 2H; H-3, H-4), $4.26\left(\mathrm{dd}, J_{6^{\prime} \mathrm{b}, 5^{\prime}}=5.1 \mathrm{~Hz}, J_{6^{\prime} \mathrm{a}, 6^{\prime} \mathrm{b}}=12.3 \mathrm{~Hz}, 1 \mathrm{H} ; \mathrm{H}-6^{\prime} \mathrm{b}\right), 4.17-$ $4.14(\mathrm{~m}, 1 \mathrm{H}, \mathrm{H}-5), 3.86\left(\mathrm{t}, J_{3^{\prime}, 2^{\prime}}=J_{3^{\prime}, 4^{\prime}}=10.0 \mathrm{~Hz}, 1 \mathrm{H} ; \mathrm{H}-3\right), 3.82$ (s, 3H, COOCH $), 3.41$ (dd, $\left.J_{2^{\prime}, 1^{\prime}}=3.6 \mathrm{~Hz}, J_{2^{\prime}, 3^{\prime}}=10.3 \mathrm{~Hz}, 1 \mathrm{H} ; \mathrm{H}-2^{\prime}\right), 2.68-2.53$ (m, 4H, $\mathrm{CH}_{2}$ (succ.)), 1.97 (s, 3H; $\mathrm{CH}_{3}(\mathrm{Ac})$. FAB-MS m/z $930\left(\mathrm{M}+\mathrm{Na}^{+}\right), \mathrm{C}_{47} \mathrm{H}_{46} \mathrm{~N}_{4} \mathrm{O}_{15}(906.89)$.

Methyl ( $\mathrm{N}$-succinimido-O-(4-O-actetyl-2-azido-6-O-benzoyl-3-O-benzyl-2-deoxy- $\alpha$-D-glucopyranosyl)-(1 $\rightarrow 4)-O$-methyl $\quad(2-O$-benzoyl-3-O-benzyl- $\alpha$-L-idopyranosyluronate)-( $\rightarrow 4)-O$ (2-azido-6-O-benzoyl-3-O-benzyl-2-deoxy- $\alpha$-D-glucopyranosyl)-(1 $\rightarrow$ 4)-(2-O-benzoyl-3-Obenzyl-1-desoxy- $\alpha$-L-idopyranosyl) uronate (12). (6.3 mg, $0.0039 \mathrm{mmol}, 5 \%) .{ }^{1} \mathrm{H}-\mathrm{NMR}(500$ $\left.\mathrm{MHz}, \mathrm{CDCl}_{3}\right) \delta=8.09-7.91\left(\mathrm{~m}, 8 \mathrm{H} ; \mathrm{Bz}, 7.55-7.11(\mathrm{~m}, 32 \mathrm{H} ; \mathrm{Ph}), 6.19\left(\mathrm{~d}, J_{1 \mathrm{a}, 2 \mathrm{a}}=9.4 \mathrm{~Hz}, 1 \mathrm{H} ; \mathrm{H}-\right.\right.$ 1a), $6.07\left(\mathrm{t}, J_{2 \mathrm{a}, 1 \mathrm{a}}=J_{2 \mathrm{a}, 3 \mathrm{a}}=8.6 \mathrm{~Hz}, 1 \mathrm{H} ; \mathrm{H}-2 \mathrm{a}\right), 5.55\left(\mathrm{~d}, J_{1 \mathrm{c}, 2 \mathrm{c}}=4.3 \mathrm{~Hz}, 1 \mathrm{H} ; \mathrm{H}-1 \mathrm{c}\right), 5.17\left(\mathrm{~d}, J_{1 \mathrm{~b}, 2 \mathrm{~b}}\right.$ $=3.4 \mathrm{~Hz}, 1 \mathrm{H} ; \mathrm{H}-1 \mathrm{~b}), 5.16\left(\mathrm{t}, J_{4 \mathrm{~d}, 5 \mathrm{~d}}=J_{4 \mathrm{~d}, 3 \mathrm{~d}}=9.8 \mathrm{~Hz}, 1 \mathrm{H} ; \mathrm{H}-4 \mathrm{~d}\right), 4.94\left(\mathrm{~d}, J_{\text {gem }}=10.6 \mathrm{~Hz}, 1 \mathrm{H}\right.$; $\left.\mathrm{CH}_{2} \mathrm{Ph}\right), 4.92\left(\mathrm{~d}, J_{\mathrm{d} 1, \mathrm{~d} 2}=3.6 \mathrm{~Hz}, 1 \mathrm{H} ; \mathrm{H}-1 \mathrm{~d}\right), 4.85-4.72\left(\mathrm{~m}, 8 \mathrm{H} ; \mathrm{CH}_{2} \mathrm{Ph}, \mathrm{H}-5 \mathrm{c}, \mathrm{H}-5 \mathrm{a}\right), 4.66(\mathrm{~d}$, $J_{\text {gem }}=10.9 \mathrm{~Hz}, 1 \mathrm{H} ; \mathrm{CH}_{2 \mathrm{Ph})}, 4.56\left(\mathrm{bd}, J_{6 \mathrm{a}, 6 \mathrm{a}^{\prime}}=11.7 \mathrm{~Hz}, 1 \mathrm{H} ; \mathrm{H}-6 \mathrm{a}\right), 4.48-4.45$ (m, 2H; H-6' d, H6b), 4.33-4.26 (m, 2H; H-3a, H-4a), 4.20-4.13 (m, 3H; H-3c, H-5b, H-6’b), 4.07 (t, $J_{4,3}=4.8$ $\mathrm{Hz}, 1 \mathrm{H} ; \mathrm{H}-4 \mathrm{c}), 4.03-4.02$ (m, 2H, H-5d, H-4b), 3.78-3.74 (m, 1H; H-3b), 3.71 (s, 3H, aCOOMe), 3.59 (t, $\left.J_{3,2}=J_{3,4}=9.6 \mathrm{~Hz}, 1 \mathrm{H} ; \mathrm{H}-3 \mathrm{~d}\right), 3.46(\mathrm{~s}, 3 \mathrm{H}, \mathrm{c}-\mathrm{COOMe}), 3.35$ (dd, $J_{2,1}=3.4$ $\left.\mathrm{Hz}, J_{2,3}=9.9 \mathrm{~Hz}, 1 \mathrm{H} ; \mathrm{H}-2 \mathrm{~b}\right), 3.32\left(\mathrm{dd}, J_{2,1}=3.6 \mathrm{~Hz}, J_{2,3}=10.2 \mathrm{~Hz}, 1 \mathrm{H} ; \mathrm{H}-2 \mathrm{~d}\right), 2.67-2.51(\mathrm{~m}$, $4 \mathrm{H}, \mathrm{CH}_{2}$ (succinimide), $1.95\left(\mathrm{~s}, 3 \mathrm{H}, \mathrm{CH}_{3}(\mathrm{Ac})\right.$. FAB-MS m/z $1654\left(\mathrm{M}+\mathrm{Na}^{+}\right), \mathrm{C}_{86} \mathrm{H}_{84} \mathrm{~N}_{6} \mathrm{O}_{27}$ (1633.61).

\section{Autocondensation of 9 (second trial)}

Compound 9 (22.3 mg, $0.026 \mathrm{mmol}$ ) was co-evaporated twice with freshly distilled toluene and dried in high vacuum for $3 \mathrm{~h}$. The dried compound was then dissolved in $\mathrm{CH}_{2} \mathrm{Cl}_{2}(1 \mathrm{ml})$ and stirred for $30 \mathrm{~min}$ in the presence of activated molecular sieves $(3 \AA \hat{)})$. After cooling to $0^{\circ} \mathrm{C}$, NIS $(6.4 \mathrm{mg}, 0.03 \mathrm{mmol})$ was added as a solid, followed by the slow addition of $50 \mu \mathrm{l}$ of a freshly prepared TMSOTf-sol. $(0.028 \mathrm{M}, 0.008 \mathrm{mmol})$. The progress of the reaction was monitored by

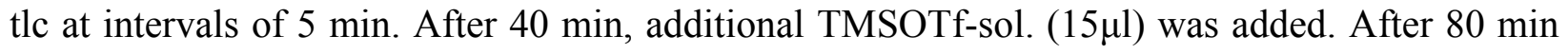
the cooling bath was removed. After $2 \mathrm{~h}$ of stirring no further change in the reaction mixture was detected; the mixture was diluted with $\mathrm{CH}_{2} \mathrm{Cl}_{2}(5 \mathrm{ml})$ and quenched by injection of sat. $\mathrm{NaHCO}_{3}$ solution $(2 \mathrm{ml})$. The separated organic phase was washed successively with sat. $\mathrm{Na}_{2} \mathrm{~S}_{2} \mathrm{O}_{3}$ and brine, dried over $\mathrm{MgSO}_{4}$ and concentrated in vacuo. The resulting residue was split into two fractions by size-exclusion chromatography (Sephadex LH-20, 1:1 $\mathrm{MeOH} / \mathrm{CH}_{2} \mathrm{Cl}_{2}$ ). The fractions containing faster running products were collected, concentrated in vacuo and acetylated with $\mathrm{Ac}_{2} \mathrm{O}$ /pyridine $2: 1$ at $0^{\circ} \mathrm{C}$ for $2 \mathrm{~h}$.

Chemical shift values for the $\mathrm{CH}_{3}$ - groups of Me-esters in the L-IdoA-residues of the proposed hexasaccharide $(5 \mathrm{mg}$, calculated average molecular weight: 1748.61 for tetra-/hexasaccharide ratio 3:1, $0.0029 \mathrm{mmol})$ and disaccharide mixture: ${ }^{1} \mathrm{H}-\mathrm{NMR}\left(500 \mathrm{MHz}, \mathrm{CDCl}_{3}\right): \delta 3.84(\mathrm{~s}, 3 \mathrm{H}$; $\mathrm{COOCH}_{3}$ disaccharide), $3.81\left(\mathrm{~s}, 3 \mathrm{H} ; \mathrm{COOCH}_{3}\right), 3.66\left(\mathrm{~s}, 3 \mathrm{H} ; \mathrm{COOCH}_{3}\right), 3.47\left(\mathrm{~s}, 3 \mathrm{H} ; \mathrm{COOCH}_{3}\right)$ 


\section{Acknowledgments}

We thank the Ministry of Science and Technology for financial support (Grant BQU 200203734) and the European Union for a fellowship (to N.-C. R) (Grant HRN-CT-200000001/Glycotrain).

\section{References}

1. De Paz, J. L.; Ojeda, R.; Reichardt, N.; Martin-Lomas, M. Eur. J. Org. Chem. 2003, 3308.

2. (a) Ojeda, R.; De Paz, J.L.; Martin-Lomas, M. Chem. Commun. 2003, 2486. (b) Ojeda, R.; Terentí, O.; De Paz, J.L.; Martin-Lomas, M. Glycoconjugate J. 2004, 21, 179.

3. (a) De Paz, J.L.; Angulo, J.; Lassaletta, J.M.; Nieto, P.M.; Redondo-Horcajo, M.; Lozano, R.M.; Gimenez-Gallego, G.; Martin-Lomas, M. ChemBiochem. 2001, 2, 673. (b) Ojeda, R.; Angulo, J.; Nieto, P.M.; Martin-Lomas, M. Can. J. Chem. 2002, 80, 673. (c) Lucas, R.; Angulo, J.; Nieto, P.M.; Martin-Lomas, M. Org. Biomol. Chem. 2003, 1, 2253. (d) De Paz, J.L.; Martín-Lomas, M. Eur. J. Org. Chem. In the press.

4. Angulo, J.; Ojeda, R.; De Paz, J.L.; Lucas, R.; Nieto, P.M.; Lozano, R.M.; Redondo-Horcajo, M.; Giménez-Gallego, G.; Martin-Lomas, M. ChemBiochem. 2004, 5, 55.

5. Klint, P.; Claesson-Welsh, L. Front. Biosci. 1999, 4, D165.

6. (a) Conrad, H.E. Heparin-Binding Proteins, Academic Press: San Diego 1998. (b) Capila, I.; Lindhardt, R.J. Angew. Chem., Int. Ed. 2002, 41, 390.

7. Casu, B.; Lindahl, U. Adv. Carbohydr. Chem. Biochem. 2001, 57, 159.

8. (a) Kochetkov, N.K. Pure \& Appl. Chem. 1975, 42, 327. (b) Kraska, U.; Micheel, F. Carbohydr. Res. 1976, 49, 195. (c) Schuerch, C. Adv. Carbohydr. Chem. Biochem. 1981, 39, 157. (d) Nakatsubo, F.; Kamitakahara, H.; Hori, M. J. Am. Chem. Soc. 1996, 118, 1677.

9. Kobayashi, S.; Morii, H.; Itoh, R.; Kimura, S.; Ohmae, M. J. Am. Chem. Soc. 2001, 123, 11825.

10. Kochetkov, N.K. Tetrahedron 1987, 43, 2389.

11. (a) Nicolaou, K.C.; Seitz, S.P.; Pavia, M.R. J. Am. Chem. Soc. 1981, 103, 1222. (b) Wetter, H.; Oertle, K. Tetrahedron Lett. 1985, 26, 5515.

12. Schmidt, R.R.; Michel, J.; Roos, M. Liebigs Ann. 1984, 1343.

13. (a) Fraser-Reid, B.; Udodong, U.E.; Wu, Z.; Ottoson, H.; Merrit, J.; Rao, C.S.; Roberts, C.; Madsen, R. Synlett 1992, 927. (b) Roberts, C.; Madsen, R.; Fraser-Reid, B. J. Am. Chem. Soc. 1995, 117, 1546.

14. Tabeur, T.; Machetto, F.; Mallet, J.M.; Duchassoy, P.; Petitou, M.; Sinaÿ, P. Carbohydr. Res. 1996, 281, 253.

15. Reichardt, N.-C. Thesis, University of Konstanz, 2004. 
16. Schmidt, R.R.; Michel, J. Angew. Chem. 1980, 92, 763; Angew. Chem. Int. Ed. 1980, 19, 731.

17. Nicolaou, K.C.; Veale, C.A.; Hwang, C.K.; Hutchison, J.; Prasad, C.V.C.; Ogilvie, W.W. Angew. Chem. 1991, 103, 304; Angew. Chem., Int. Ed. 1991, 30, 299.

18. (a) Soll, R.M.; Steitz, S.P. Tetrahedron Lett. 1987, 28, 5457. (b) Abbas, S.A.; Haines, A.H. Carbohydr. Res. 1975, 39, 358.

19. Konradsson, P.; Mootoo, D.R.; McDevitt, R.E.; Fraser-Reid, B. Chem. Commun. 1990, 370.

20. Höfle, G.; Steglitz, W.; Vorbrüggen, H. Angew. Chem. 1978, 90, 602.

21. (a) Zhang, Z.; Ollman, I.R.; Ye, X.-S.; Wischnat, R.; Baasov, T.; Wong, C.-H. J. Am. Chem. Soc. 1999, 121, 734. (b) Thiering, S.; Sowa, C.E.; Thiem, J. J. Chem. Soc., Perkin Trans. 1 2001, 8, 801 .

22. Vogel, C.; Steffan, W.; Boye, H.; Kirsten, H.; Betaneli, V.I.; Ott, A.Y.; Kochetkov, N.K. Carbohydr. Res. 1992, 237, 131. 